\title{
Effect of regional muscle damage and inflammation \\ following eccentric exercise on electrical resistance and the body composition assessment using bioimpedance spectroscopy
}

\author{
Keisuke Shiose ${ }^{1,2}$ [D $\cdot$ Yoko Tanabe $^{3} \cdot$ Takahiro Ohnishi $^{3} \cdot$ Hideyuki Takahashi $^{3}$
}

Received: 25 February 2019 / Accepted: 30 July 2019 / Published online: 6 August 2019

(c) The Physiological Society of Japan and Springer Japan KK, part of Springer Nature 2019

\begin{abstract}
We investigated the effect of muscle damage and inflammation on electrical resistance and the body composition assessment by using bioimpedance spectroscopy (BIS). Twenty-two subjects completed 30 repetitions of maximal eccentric contractions of the elbow flexors with one arm. Whole-body resistance of extracellular and intracellular components $\left(R_{\mathrm{e}}\right.$ and $R_{\mathrm{i}}$, respectively) on the exercised and non-exercised sides were measured using BIS. Body composition was calculated from both sides of resistance at baseline and $96 \mathrm{~h}$ after exercise. $R_{\mathrm{e}}$ decreased only on the exercised side at $96 \mathrm{~h}$ after exercise $(P<0.05)$. Fat-free and fat mass values estimated from resistance on the exercised side were altered by $3.1 \%$ and $-15.6 \%$, respectively, at $96 \mathrm{~h}$ after exercise $(P<0.05)$; those estimated from the non-exercised side were unaltered. Eccentric exerciseinduced muscle damage and inflammation reduce $R_{\mathrm{e}}$ and induce non-negligible estimation error in the body composition assessment using BIS.
\end{abstract}

Keywords Electrical resistance $\cdot$ Muscle damage $\cdot$ Inflammation $\cdot$ Body composition

\section{Introduction}

Bioimpedance spectroscopy (BIS) is an accessible method for estimating body composition [i.e., fat mass (FM), fatfree mass (FFM), and body water content]. BIS impresses multi-frequency current to the whole body or each body segment, and body composition was estimated using theoretical and/or empirical estimation equation with measured electrical properties, for instance, reactance $\left(X_{\mathrm{c}}\right)$, resistance $(R)$, and impedance $(Z)$. Previous studies have shown a relatively wide limit of agreement between body composition measured by BIS and dual-energy X-ray absorptiometry; however, estimated values were highly correlated and the mean of difference between methods is small in healthy

Keisuke Shiose

kshiose@cc.miyazaki-u.ac.jp

1 Faculty of Education, University of Miyazaki, 1-1 Gakuen Kibanadai-nishi, Miyazaki 889-2192, Japan

2 Fukuoka University Institute for Physical Activity, 8-19-1 Nanakuma, Jonan-ku, Fukuoka 814-0180, Japan

3 Japan Institute of Sports Sciences, 3-15-1 Nishigaoka, Kita-ku, Tokyo 115-0056, Japan pediatric, adult, and elderly subjects [1-3]. In a study of athletes, BIS was reported to have good accuracy for estimating body water content compared with the isotope dilution method [4]. Additionally, another study suggested that body composition was accurately estimated in trained Caucasians when air-displacement plethysmography and BIS were concomitantly used [5]. Therefore, there is still room for improvement in the accuracy for individual use [6], but some evidence supports the use of BIS in body composition analysis of athletes.

Notably, athletes regularly have exercise-induced inflammation in any body segment and/or their whole body during daily training. Physiological responses to eccentric exerciseinduced muscle damage and inflammation are typified by a decrease in maximal voluntary torque and range of motion [7], presence of muscle soreness [7, 8], and an increase in blood concentrations of muscle proteins and inflammatory markers [8-10]. Regional swelling and an increase in the muscle cross-sectional area have been also reported based on magnetic resonance imaging (MRI) and a measurement of the transverse relaxation time [11].

A change in the regional hydration status accompanied by regional muscle damage and inflammation may cause a large estimation error because the equation for estimating body 
composition using BIS generally applies to the assumption that hydration status is constant (i.e., water volume/FFM of approximately 0.73 ) and uniform between each body segment. A previous study using MRI and single-frequency bioimpedance analysis showed that the electrical properties (e.g., resistance and reactance) of the injured muscle were altered after muscle injury, which was categorized as grades I-III [12, 13], but change in whole-body resistance and the effect on body composition estimation were not revealed. To estimate body composition in athletes accurately, it is important to understand how regional muscle damage and inflammation affect body composition estimation using BIS.

The main objective of this research was to determine the effect of muscle damage following eccentric exercise of the elbow flexors on body electrical resistance and the body composition estimation using BIS. We hypothesized that muscle damage following eccentric exercise would regiospecifically decrease extracellular resistance because of swelling. In addition, a regiospecific increase in body water would cause overestimation of FFM when using a wholebody model of BIS.

\section{Methods}

\section{Subjects}

Twenty-two male subjects (age, $28.8 \pm 3.7$ years; height, $172.0 \pm 5.1 \mathrm{~cm}$; body mass, $68.8 \pm 10.3 \mathrm{~kg}$; body mass index, $23.3 \pm 3.6 \mathrm{~kg} / \mathrm{m}^{2}$ ) participated in this study. Written informed consent was obtained from all participants, and the study procedure was approved by the ethical committee of the Japan Institute of Sports Sciences.

\section{Experimental procedures}

Subjects completed the elbow flexion eccentric exercise using one arm in accordance with a previous study [14]. They occasionally participated in recreational sports (e.g., running, cycling, resistance training, and soccer), but did not perform regular training. The exercised arm was determined randomly without reference to the dominant side. According to the manufacturer's guidelines, Biodex system (System 4; Biodex Medical System, New York City, NY, USA) was used for exercise following calibration. Participants were strapped to a dynamometer chair with their arm resting on the armrest. The fully extended elbow joint angle was determined as $0^{\circ}$, and range of motion was set from $130^{\circ}$ to $10^{\circ}$. Following familiarization sessions, participants performed 30 repetitions of maximal eccentric exercise of the elbow flexors at $120 \% \mathrm{~s}$ with a rest period of $12 \mathrm{~s}$ between contractions. Participants were instructed to complete each exercise with full effort.
Body resistance, body composition, arm circumference, and blood samples were obtained immediately before exercise and at 24, 48, 72, and $96 \mathrm{~h}$ after exercise (days 0, 1, 2, 3, and 4, respectively). Participants were instructed to maintain a normal dietary habit. Vigorous exercise and consumption of an alcoholic drink were restricted during the experiment.

\section{Bioimpedance spectroscopy measurement}

Body composition was measured using the BIS system (SFB7; ImpediMed, Pinkenba, QLD, Australia). Electrodes (Red Dot; 3M Health Care, St. Paul, MN, USA) were placed bilaterally on the exercised and non-exercised sides as injection and sensing electrodes. Injection electrodes were placed on the dorsal surface of the hands and feet proximal to the metacarpal-phalangeal and metatarsal-phalangeal joints. Sensing electrodes were placed on the middorsum of the wrist, centered on a line joining the bony prominences of the radius and ulna; the lateral epicondyle of the humerus; tip of the acromion; greater trochanter of the femur, and midanterior ankle, centered on a line joining the malleolus lateralis and malleolus medialis of the upper limb, trunk, and lower limb. The electrode position was marked and standardized for each measurement. Body resistance was measured for the exercised and non-exercised sides of the body for the whole body (wrist-to-ankle model) and the forearm, upper arm, trunk, and leg segments. Measurements were conducted with participants in the standing position while in a temperaturecontrolled room $\left(21-22{ }^{\circ} \mathrm{C}\right)$.

Resistance values of extracellular and intracellular components ( $R_{\mathrm{e}}$ and $R_{\mathrm{i}}$, respectively) were based on a Cole plot using ImpediMed SFB7 Multi-Frequency Analysis software (ImpediMed, Pinkenba). The analytical setting was set up in accordance with that in previous studies $[15,16]$. In the whole-body model of BIS, FM, FFM, extracellular and intracellular water (ECW and ICW, respectively) content, and total body water (TBW) content were calculated from wristto-ankle resistance using a manufacturer-provided equation based on the Hanai mixture theory $[17,18]$. In the segmental model, the impedance index of the extracellular $\left(L^{2} / R_{\mathrm{e}}\right)$ and intracellular $\left(L^{2} / R_{\mathrm{j}}\right)$ components was calculated as relative indicators of ECW and ICW contents, where $L$ is the length $(\mathrm{cm})$ of each segment.

\section{Upper arm circumference}

The upper arm circumference was measured using an MRI system (Magnetom Skyra, Verio; Siemens, Erlangen, Germany). Cross-sectional images of the area 60\% distal to the length between the acromial process and epicondylus lateralis humeri were obtained using a body array and spine array coil. An area-measuring program (ISIS, Hitachi 
Medical Corporation, Tokyo, Japan) was used to determine circumference.

\section{Blood analysis}

Blood samples were taken from the antecubital vein using vacuum tubes. Samples were centrifuged at $3000 \times g$ for $10 \mathrm{~min}$ and stored at $4{ }^{\circ} \mathrm{C}$ until analysis. Serum creatine kinase $(\mathrm{CK})$ was measured in an independent laboratory (LSI Medience Corporation, Tokyo, Japan).

\section{Statistical analysis}

All data are presented as a mean \pm standard deviation. Data of the serum CK on day 1 and arm circumference were obtained from 21 and 16 participants, respectively, in whom they could be measured. Two-factor factorial repeated-measure analysis of variance (ANOVA) was used to detect differences in data between the exercised and non-exercised sides. One-way repeated-measure ANOVA was used to detect changes in the serum CK level and upper arm circumference. When a significant interaction was identified, data were subsequently analyzed using the Bonferroni post hoc test. Statistical significance was set at $P<0.05$. Statistical calculations were performed using SPSS Statistics version 24 (IBM Corp., Armonk, NY, USA).
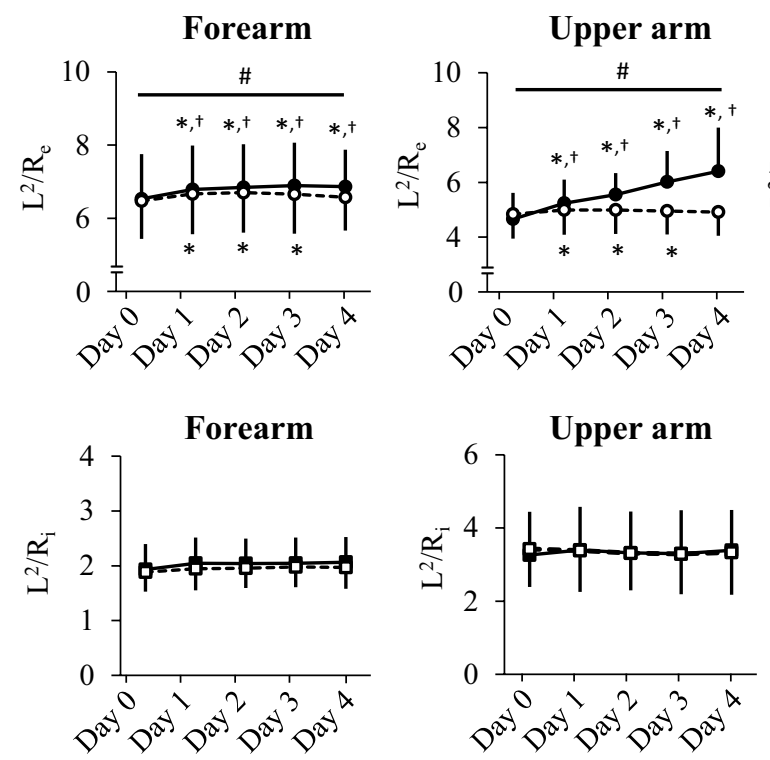

Fig. 1 Changes in impedance indexes in each segment. Values are presented as mean \pm standard deviation. Open circles or square with dotted line, non-exercised side; dark circles or square with solid line, exercised side. ${ }^{\#} P<0.05$, interaction for time and condition.

\section{Results}

\section{Serum creatine kinase level}

Serum CK levels increased with each day after exercise (main effect for time $P<0.001$ ). Serum CK levels at day 1 and day 2 were not significantly different compared with those on day 0 (day $0: 185 \pm 267$, day $1: 2152 \pm 4392$, and day 2: $6563 \pm 11892$ IU/L). However, serum CK levels on day 3 and day 4 were significantly increased compared with the baseline value (day 3: $13495 \pm 15588$ and day 4: 16 $475 \pm 14850 \mathrm{IU} / \mathrm{L}$; both $P<0.01$ vs day 0$)$.

\section{Upper arm circumference}

Upper arm circumference of the exercised side was $26.3 \pm 2.4 \mathrm{~cm}$ on day 0 . It continued to increase after exercise compared to the baseline value (day $1: 26.9 \pm 2.2$, day 2 : $27.0 \pm 2.2$, day 3: $27.5 \pm 2.1$, and day $4: 27.7 \pm 2.2 \mathrm{~cm}$; main effect for time $P<0.001$, all $P<0.05$ vs day 0 ).

\section{Segmental resistance and water status}

On day $0, L^{2} / R_{\mathrm{e}}$ and $L^{2} / R_{\mathrm{i}}$ in the forearm, upper arm, trunk, and leg were similar between the exercised and nonexercised sides. The $L^{2} / R_{\mathrm{e}}$ in the forearm and upper arm increased in the exercised side with each day after exercise and was higher than that in the non-exercised side (Fig. 1).
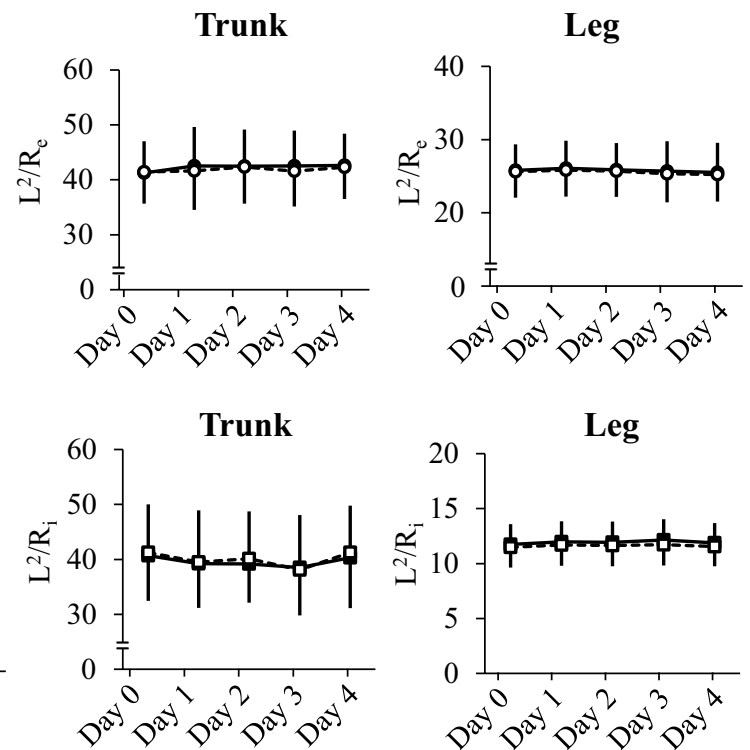

$* P<0.05$, from day 0 in the same condition. ${ }^{\dagger} P<0.05$, between conditions at the same time. $L^{2} / R_{e}$ impedance index of the extracellular components, $L^{2} / R_{i}$ impedance index of the intracellular components 
Segmental resistance values and impedance indexes at day 0 and day 4 are shown in Table 1. Significant correlations were found between percent changes from day 0 to day 4 of the $L^{2} / R_{\mathrm{e}}$ in the upper arm and serum CK level, and $L^{2} / R_{\mathrm{e}}$ and upper arm circumference (Fig. 2a, b).

\section{Wrist-to-ankle resistance and whole-body composition}

Wrist-to-ankle $R_{\mathrm{e}}$ and $R_{\mathrm{i}}$ were similar between the nonexercised and exercised sides of the body on day 0 (nonexercised side $578 \pm 68$ and $1194 \pm 226$; exercised side $582 \pm 74$ and $1195 \pm 253$, respectively). There was a significant interaction for time and condition for wrist-to-ankle $R_{\mathrm{e}}(P<0.001)$. In the exercised side, wrist-to-ankle $R_{\mathrm{e}}$ decreased with each day after exercise (day 1,556 \pm 67 ; day 2, 550 \pm 62 ; day $3,544 \pm 64$; day $4,540 \pm 59$; all $P<0.05$ vs day 0 ). In the non-exercised side, wrist-to-ankle $R_{\mathrm{e}}$ decreased at day $1(568 \pm 66 ; P=0.044)$, but those at days 2,3 , and 4 were similar to day $0(566 \pm 65,574 \pm 69,575 \pm 63$, respectively; all $P>0.05$ ). Wrist-to-ankle $R_{\mathrm{i}}$ was not changed in the non-exercised and exercised sides (interaction for time and condition, $P=0.148$; main effect for time, $P=0.354$; main effect for condition, $P=0.504)$. Whole-body compositions estimated from the wrist-to-ankle resistance of the non-exercised and exercised sides at day 0 and day 4 are shown in Table 2 . On day 0 , body composition parameters estimated from the non-exercised and exercised sides of the body were similar. At day 4, FFM, ECW, ICW, and TBW values estimated from the exercised side were larger, and FM estimated from the exercised side was smaller than that estimated from the non-exercised side (interaction for time and condition, both $P<0.05$ ). There was no change in body mass from day 0 to day $4(68.8 \pm 10.3$ to $68.9 \pm 10.2 \mathrm{~kg}$; main effect for time $P=0.425)$.

\section{Discussion}

In this study, we demonstrated the effect of eccentric exercise-induced regional muscle damage and inflammation on body resistance and body composition analysis using BIS. Our results clearly showed that extracellular resistance of the arm decreased after eccentric exercise of the elbow flexors in one arm. Consequently, FFM and FM values estimated from the non-exercised and exercised sides using BIS varied widely. Hence, our hypotheses are verified by our findings.
Table 1 Non-exercised and exercised side of body resistance and impedance index in each segment

\begin{tabular}{|c|c|c|c|c|c|}
\hline & \multicolumn{2}{|c|}{ Non-exercised side } & \multicolumn{2}{|l|}{ Exercised side } & \multirow{2}{*}{$\begin{array}{l}\text { Interaction } \\
P \text { value }\end{array}$} \\
\hline & Day 0 & Day 4 & Day 0 & Day 4 & \\
\hline \multicolumn{6}{|l|}{ Forearm } \\
\hline$R_{\mathrm{e}}(\Omega)$ & $156 \pm 20$ & $153 \pm 17$ & $156 \pm 23$ & $147 \pm 17 *, \dagger$ & 0.004 \\
\hline$R_{\mathrm{i}}(\Omega)$ & $541 \pm 96$ & $519 \pm 96$ & $542 \pm 130$ & $502 \pm 103$ & 0.207 \\
\hline$L^{2} / R_{\mathrm{e}}$ & $6.48 \pm 1.04$ & $6.57 \pm 0.91$ & $6.53 \pm 1.22$ & $6.87 \pm 1.01 *, \dagger$ & 0.006 \\
\hline$L^{2} / R_{\mathrm{i}}$ & $1.89 \pm 0.36$ & $1.97 \pm 0.39$ & $1.93 \pm 0.47$ & $2.06 \pm 0.46$ & 0.405 \\
\hline \multicolumn{6}{|c|}{ Upper arm } \\
\hline$R_{\mathrm{e}}(\Omega)$ & $120 \pm 19$ & $118 \pm 18$ & $124 \pm 22$ & $91 \pm 17 *, \dagger$ & $<0.001$ \\
\hline$R_{\mathrm{i}}(\Omega)$ & $181 \pm 56$ & $186 \pm 57$ & $188 \pm 61$ & $187 \pm 72$ & 0.120 \\
\hline$L^{2} / R_{\mathrm{e}}$ & $4.84 \pm 0.90$ & $4.91 \pm 0.86$ & $4.66 \pm 0.96$ & $6.40 \pm 1.59 * \dagger$ & $<0.001$ \\
\hline$L^{2} / R_{\mathrm{i}}$ & $3.43 \pm 1.18$ & $3.34 \pm 1.10$ & $3.27 \pm 1.04$ & $3.39 \pm 1.16$ & 0.145 \\
\hline \multicolumn{6}{|l|}{ Trunk } \\
\hline$R_{\mathrm{e}}(\Omega)$ & $68 \pm 8$ & $66 \pm 7$ & $68 \pm 8$ & $66 \pm 7$ & 0.582 \\
\hline$R_{\mathrm{i}}(\Omega)$ & $70 \pm 15$ & $70 \pm 15$ & $73 \pm 24$ & $73 \pm 18$ & 0.919 \\
\hline$L^{2} / R_{\mathrm{e}}$ & $41.44 \pm 6.03$ & $42.30 \pm 5.54$ & $41.23 \pm 5.79$ & $42.64 \pm 5.76$ & 0.305 \\
\hline$L^{2} / R_{\mathrm{i}}$ & $41.24 \pm 8.81$ & $41.27 \pm 10.15$ & $40.67 \pm 9.35$ & $40.30 \pm 9.50$ & 0.883 \\
\hline \multicolumn{6}{|l|}{ Leg } \\
\hline$R_{\mathrm{e}}(\Omega)$ & $234 \pm 26$ & $238 \pm 28$ & $233 \pm 28$ & $237 \pm 30$ & 0.373 \\
\hline$R_{\mathrm{i}}(\Omega)$ & $529 \pm 98$ & $525 \pm 93$ & $521 \pm 105$ & $515 \pm 101$ & 0.502 \\
\hline$L^{2} / R_{\mathrm{e}}$ & $25.62 \pm 3.54$ & $25.23 \pm 3.68$ & $25.81 \pm 3.55$ & $25.54 \pm 4.01$ & 0.466 \\
\hline$L^{2} / R_{\mathrm{i}}$ & $11.49 \pm 1.85$ & $11.55 \pm 1.80$ & $11.75 \pm 2.14$ & $11.88 \pm 2.09$ & 0.496 \\
\hline
\end{tabular}

Values are presented as mean \pm standard deviation. $* P<0.05$, from day 0 in the same condition. ${ }^{\dagger} P<0.05$, between conditions at the same time

$R_{e}$ resistance of extracellular components, $R_{i}$ resistance of intracellular components, $L^{2} / R_{e}$ impedance index of the extracellular components, $L^{2} / R_{i}$ impedance index of the intracellular components 

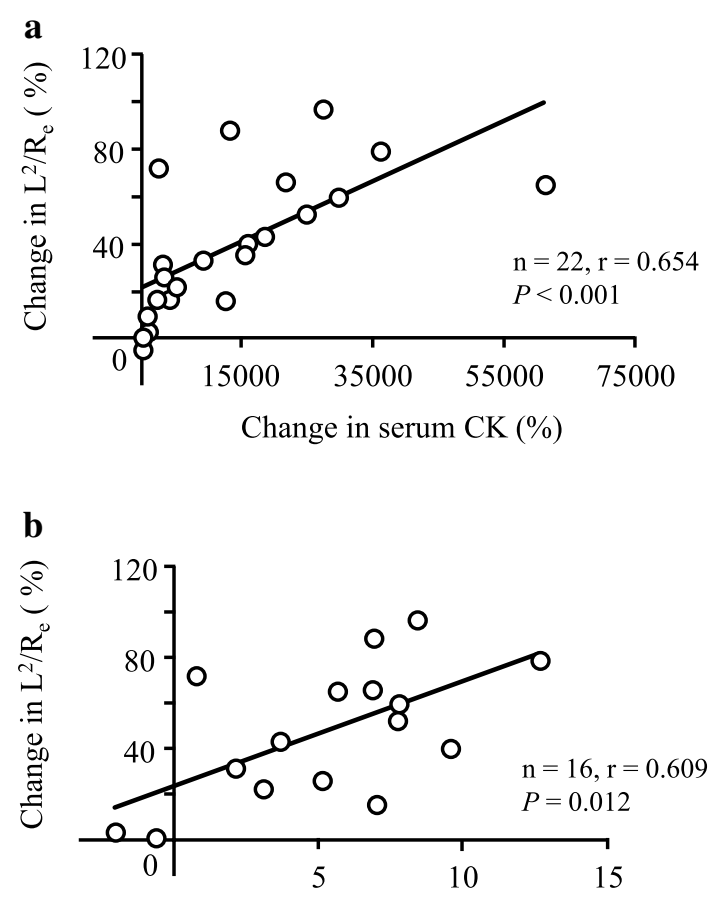

Change in upper arm circumference (\%)

Fig. 2 Relationship between the a extracellular impedance index $\left(L^{2} /\right.$ $R_{\mathrm{e}}$ ) and serum creatine kinase (CK) level, and $\mathbf{b} L^{2} / R_{\mathrm{e}}$ and upper arm circumference of the upper arm on the exercised side

Regional muscle damage was induced by eccentric exercise of the elbow flexors in this study, and the serum CK level obviously increased 3 days after exercise. This finding agrees with the results of previous studies in which a similar muscle damage model was used [7, 19]. Technically, it is difficult to determine the site of original inflammation; however, this response probably originated from the segment that underwent eccentric exercise since participants were prohibited from performing any exercise, except for daily activity during the intervention period.

Our data showed that change in resistance occurs regiospecifically and is attributed to extracellular components in a multi-segment model of BIS. These results were complementary to those reported in a previous study [12, 13]; the authors reported change in resistance at $50 \mathrm{kHz}$ of current, which is reflected by both intracellular and extracellular resistances, only in the injured segment. One possible cause of the induced change in $R_{\mathrm{e}}$ is swelling. Following muscle damage, vasodilatation and increased permeability of the blood vessels occurs because of the effects of some substrates (e.g., histamine and kinin); as a result, swelling is induced in the damaged site [20,21]. Principally, in BIS, an increase in tissue water content is linked to a decrease in resistance, and water flux with swelling appears likely to be reflected by $R_{\mathrm{e}}$ [22]. The suggestion that swelling is an inflammatory response that causes a decrease in resistance is partly supported by our results that change in the impedance index of the extracellular component correlates with change in the serum CK level and upper arm circumference, which are main indicators of inflammation and swelling.

Four days after the eccentric exercise, FFM and FM estimated from the wrist-to-ankle resistance of the exercised side were found to have changed by approximately $1.8 \mathrm{~kg}$ (Table 2). This assessment must include some errors because the study period was too short for body composition to be altered. In addition, our results showed that body composition estimated from the non-exercised side and body weight remained static. The segment model of BIS suggested that only about $100 \mathrm{~mL}$ of ECW was accumulated in the exercised arm in that the ECW content could be estimated by multiplying $L^{2} / R_{\mathrm{e}}$ by the assumed rho value, which is generally 47 for men [23,24]. A previous study demonstrated that the total body resistance values were 52\% for the arm (34\% for the forearm and $18 \%$ for the upper arm), $4 \%$ for the trunk, and $44 \%$ for the leg of Caucasian adults [25]. Similarly, our data of Japanese young adults showed that arm resistance was more than $40 \%$ of the total body resistance. Accordingly, it can be interpreted that a decrease in arm resistance is caused by a small absolute amount of water accumulation after eccentric exercise, however it is excessively reflected by an increase in FFM (and compensatory decrease in FM) in the whole-body model due to the characteristic of resistance distribution and assumption about hydration of FFM.
Table 2 Whole-body composition estimated from the wrist-to-ankle resistance of the non-exercised and exercised sides

\begin{tabular}{|c|c|c|c|c|c|c|c|}
\hline & \multicolumn{3}{|c|}{ Non-exercised side } & \multicolumn{3}{|c|}{ Exercised side } & \multirow{2}{*}{$\begin{array}{l}\text { Interaction } \\
P \text {-value }\end{array}$} \\
\hline & Day 0 & Day 4 & & Day 0 & Day 4 & & \\
\hline Fat-free mass (kg) & $57.8 \pm 8.7$ & $58.0 \pm 8.5$ & $(-0.3)$ & $57.8 \pm 8.8$ & $59.6 \pm 8.2^{* \dagger}$ & (3.1) & $<0.001$ \\
\hline Fat mass (kg) & $11.0 \pm 4.1$ & $10.9 \pm 4.0$ & $(-0.7)$ & $11.0 \pm 4.4$ & $9.3 \pm 4.7 *, \dagger$ & $(-15.6)$ & $<0.001$ \\
\hline $\mathrm{ECW}(\mathrm{L})$ & $18.2 \pm 2.6$ & $18.2 \pm 2.5$ & $(0.2)$ & $18.1 \pm 2.7$ & $19.0 \pm 2.4^{*}$, & (4.7) & $<0.001$ \\
\hline ICW (L) & $24.2 \pm 3.9$ & $24.2 \pm 3.9$ & $(0.4)$ & $24.2 \pm 3.9$ & $24.6 \pm 3.7^{*}$ & (2.0) & 0.011 \\
\hline TBW (L) & $42.3 \pm 6.4$ & $42.5 \pm 6.2$ & $(0.3)$ & $42.3 \pm 6.4$ & $43.6 \pm 6.0^{*}, \dagger$ & (3.1) & $<0.001$ \\
\hline
\end{tabular}

Values are presented as mean \pm standard deviation. The average rate of change (\%) is noted in brackets. $* P<0.05$, from day 0 in the same condition. ${ }^{\dagger} P<0.05$, between conditions at the same time

$E C W$ extracellular water, $I C W$ intracellular water, $T B W$ total body water 
For athletes, the fact that regional exercise-induced muscle damage and inflammation impair body composition estimation using BIS deserves more attention since these individuals are prone to experiencing muscle damage in daily training. Our data strongly suggest that the current-carrying area should be determined to exclude damaged segments.

There are some limitations in this study. First, BIS can measure $R_{\mathrm{e}}$ and $R_{\mathrm{i}}$ separately because the cell membrane acts as a capacitor. However, since the muscle cells may have structural damage after eccentric exercise [26], it is unclear whether cellular resistance is correctly discriminated. If the cell membrane does not act as a capacitor, an increase in $R_{\mathrm{e}}$ and a compensatory decrease in $R_{\mathrm{i}}$ coinstantaneously occur in principle. However, the exercise side-specific behavior of $R_{\mathrm{i}}$ was not observed in this study. Second, we used an equation based on the Hanai mixture theory and Cole plot to estimate body composition. Because assumed rho values were applied in the estimation equation we used here, if structural characteristics of body water (i.e., "free water" or "bond water" [27]) were altered with muscle damage and inflammation, body composition calculation includes additional errors. In addition, several other estimation equations have been proposed in previous studies [28]. It should be understood that the effect of muscle damage and inflammation on the body composition analysis depends on the applied estimation equation. Third, because of body resistance distribution, we interpreted that each body segment should have a different effect on muscle damage-induced change of resistance in the body composition assessment. Similarly, the effect on body composition analysis depends on whether muscle damage and inflammation occur in the dominant or non-dominant side in that body resistance at the dominant side differs from that in the non-dominant side [29]. To assess the body composition accurately using BIS, future study to confirm the above problems should be conducted gradually.

\section{Conclusions}

Muscle damage and inflammation following eccentric exercise of the elbow flexors induce regiospecific reduction in extracellular resistance. Because of resistance distribution in the body, even if muscle damage occurs in a small segment of the body, non-negligible estimation error is caused in the body composition assessment when using a wholebody model of BIS.

Acknowledgements We gratefully acknowledge all the participants for dedicating their time and effort in this study, and the staff of the Japan Institute of Sports Sciences for providing technical support.

Author contributions KS, YT, TO, and HT conceived and designed the research; KS, YT, TO, and HT performed the experiments; KS and
YT analyzed the data; KS, YT, TO, and HT interpreted the results of the experiments; KS prepared the figures; KS drafted the manuscript; KS, YT, TO, and HT revised the manuscript; and KS, YT, TO, and HT read and approved the final manuscript.

Funding No funding was received for this project.

\section{Compliance with ethical standards}

Conflict of interest The authors declare that they have no conflict of interest.

Ethical approval All procedures performed in studies involving human participants were in accordance with the ethical standards of the institutional research committee and with the 1964 Helsinki Declaration and its later amendments or comparable standards.

Informed consent Informed consent was obtained from all individual participants included in the study.

\section{References}

1. Fors H, Gelander L, Bjarnason R, Albertsson-Wikland K, Bosaeus I (2002) Body composition, as assessed by bioelectrical impedance spectroscopy and dual-energy X-ray absorptiometry, in a healthy paediatric population. Acta Paediatr 91:755-760

2. Tengvall M, Ellegard L, Malmros V, Bosaeus N, Lissner L, Bosaeus I (2009) Body composition in the elderly: reference values and bioelectrical impedance spectroscopy to predict total body skeletal muscle mass. Clin Nutr 28:52-58. https://doi. org/10.1016/j.clnu.2008.10.005

3. Shafer KJ, Siders WA, Johnson LK, Lukaski HC (2009) Validity of segmental multiple-frequency bioelectrical impedance analysis to estimate body composition of adults across a range of body mass indexes. Nutrition 25:25-32. https://doi.org/10.1016/j. nut.2008.07.004

4. Quiterio AL, Silva AM, Minderico CS, Carnero EA, Fields DA, Sardinha LB (2009) Total body water measurements in adolescent athletes: a comparison of six field methods with deuterium dilution. J Strength Cond Res 23:1225-1237. https://doi.org/10.1519/ JSC.0b013e3181a9ec39

5. Kerr A, Slater G, Byrne N, Chaseling J (2015) Validation of bioelectrical impedance spectroscopy to measure total body water in resistance-trained males. Int J Sport Nutr Exerc Metab 25:494503. https://doi.org/10.1123/ijsnem.2014-0188

6. Ackland TR, Lohman TG, Sundgot-Borgen J, Maughan RJ, Meyer NL, Stewart AD, Muller W (2012) Current status of body composition assessment in sport: review and position statement on behalf of the ad hoc research working group on body composition health and performance, under the auspices of the I.O.C. Med Commiss Sports Med 42:227-249. https://doi.org/10.2165/11597140-00000 0000-00000

7. Tanabe Y, Maeda S, Akazawa N, Zempo-Miyaki A, Choi Y, Ra SG, Imaizumi A, Otsuka Y, Nosaka K (2015) Attenuation of indirect markers of eccentric exercise-induced muscle damage by curcumin. Eur J Appl Physiol 115:1949-1957. https://doi. org/10.1007/s00421-015-3170-4

8. Silva LA, Pinho CA, Silveira PC, Tuon T, De Souza CT, DalPizzol F, Pinho RA (2010) Vitamin E supplementation decreases muscular and oxidative damage but not inflammatory response induced by eccentric contraction. J Physiol Sci 60:51-57. https:// doi.org/10.1007/s12576-009-0065-3 
9. Nosaka K, Clarkson PM, Apple FS (1992) Time course of serum protein changes after strenuous exercise of the forearm flexors. $\mathrm{J}$ Lab Clin Med 119:183-188

10. MacIntyre DL, Sorichter S, Mair J, Berg A, McKenzie DC (2001) Markers of inflammation and myofibrillar proteins following eccentric exercise in humans. Eur J Appl Physiol 84:180-186. https://doi.org/10.1007/s004210170002

11. Takahashi H, Kuno S, Miyamoto T, Yoshioka H, Inaki M, Akima H, Katsuta S, Anno I, Itai Y (1994) Changes in magnetic resonance images in human skeletal muscle after eccentric exercise. Eur J Appl Physiol Occup Physiol 69:408-413

12. Nescolarde L, Yanguas J, Lukaski H, Alomar X, RosellFerrer J, Rodas G (2013) Localized bioimpedance to assess muscle injury. Physiol Meas 34:237-245. https://doi. org/10.1088/0967-3334/34/2/237

13. Nescolarde L, Yanguas J, Lukaski H, Alomar X, Rosell-Ferrer J, Rodas G (2015) Effects of muscle injury severity on localized bioimpedance measurements. Physiol Meas 36:27-42. https://doi. org/10.1088/0967-3334/36/1/27

14. Tanabe Y, Chino K, Ohnishi T, Ozawa H, Sagayama H, Maeda S, Takahashi H (2018) Effects of oral curcumin ingested before or after eccentric exercise on markers of muscle damage and inflammation. Scand J Med Sci Sports. https://doi.org/10.1111/ sms. 13373

15. Yamada Y, Ikenaga M, Takeda N, Morimura K, Miyoshi N, Kiyonaga A, Kimura M, Higaki Y, Tanaka H (2014) Estimation of thigh muscle cross-sectional area by single- and multifrequency segmental bioelectrical impedance analysis in the elderly. J Appl Physiol 116:176-182. https://doi.org/10.1152/japplphysiol.00772 .2013

16. Shiose K, Yamada Y, Motonaga K, Sagayama H, Higaki Y, Tanaka H, Takahashi H (2016) Segmental extracellular and intracellular water distribution and muscle glycogen after 72-h carbohydrate loading using spectroscopic techniques. J Appl Physiol 121:205-211. https://doi.org/10.1152/japplphysiol.00126.2016

17. De Lorenzo A, Andreoli A, Matthie J, Withers P (1997) Predicting body cell mass with bioimpedance by using theoretical methods: a technological review. J Appl Physiol 82:1542-1558. https://doi. org/10.1152/jappl.1997.82.5.1542

18. Hanai T (1968) Electrical properties of emulsions. In: Sherman PH (ed) Emulsion science. Academic Press, London, pp 354-477

19. Clarkson PM, Nosaka K, Braun B (1992) Muscle function after exercise-induced muscle damage and rapid adaptation. Med Sci Sports Exerc 24:512-520

20. Clarkson PM, Sayers SP (1999) Etiology of exercise-induced muscle damage. Can J Appl Physiol 24:234-248
21. Tortora GJ, Derrickson B (2009) The lymphatic system and immunity. Principles of anatomy and physiology, 12th edn. Wiley, New York

22. Shiose K, Yamada Y, Motonaga K, Takahashi H (2017) Circadian variation of extracellular and intracellular resistance of the leg, arm, and trunk in healthy humans: a segmental bioimpedance spectroscopy study. Biomed Phys Eng Express 3:065007

23. Yamada Y, Schoeller DA, Nakamura E, Morimoto T, Kimura M, Oda $S$ (2010) Extracellular water may mask actual muscle atrophy during aging. J Gerontol A Biol Sci Med Sci 65:510-516. https:// doi.org/10.1093/gerona/glq001

24. Zhu F, Kuhlmann MK, Kaysen GA, Sarkar S, Kaitwatcharachai C, Khilnani R, Stevens L, Leonard EF, Wang J, Heymsfield S, Levin NW (2006) Segment-specific resistivity improves body fluid volume estimates from bioimpedance spectroscopy in hemodialysis patients. J Appl Physiol 100:717-724. https://doi.org/10.1152/ japplphysiol.00669.2005

25. Organ LW, Bradham GB, Gore DT, Lozier SL (1994) Segmental bioelectrical impedance analysis: theory and application of a new technique. J Appl Physiol 77:98-112. https://doi.org/10.1152/ jappl.1994.77.1.98

26. Proske U, Morgan DL (2001) Muscle damage from eccentric exercise: mechanism, mechanical signs, adaptation and clinical applications. J Physiol 537:333-345. https://doi.org/10.111 1/j.1469-7793.2001.00333.x

27. Brooks J (1934) Bound water in muscle. J Gen Physiol 17:783-790

28. Kyle UG, Bosaeus I, De Lorenzo AD, Deurenberg P, Elia M, Gómez JM, Heitmann BL, Kent-Smith L, Melchior J-C, Pirlich M, Scharfetter H, Schols AM, Pichard C, Composition of the ESPEN Working Group (2004) Bioelectrical impedance analysis-part I: review of principles and methods. Clin Nutr 23:1226-1243. https ://doi.org/10.1016/j.clnu.2004.06.004

29. Ward LC, Isenring E, Dyer JM, Kagawa M, Essex T (2015) Resistivity coefficients for body composition analysis using bioimpedance spectroscopy: effects of body dominance and mixture theory algorithm. Physiol Meas 36:1529-1549. https://doi. org/10.1088/0967-3334/36/7/1529

Publisher's Note Springer Nature remains neutral with regard to jurisdictional claims in published maps and institutional affiliations. 